\title{
Emergency in False-Electrical Storm in Patients with Implanted Cardioverter Defibrillator
}

\author{
Maurizio Santomauro ${ }^{*}$, Mario Petretta ${ }^{3}$, Carla Riganti ${ }^{2}$, Francesco Elia ${ }^{1}$, Riccardo Franco ${ }^{1}$, Francesco Cacciatore ${ }^{1}$, Mario Alberto \\ Santomauro ${ }^{1}$ and Domenico Bonaduce ${ }^{3}$ \\ ${ }^{1 *}$ Department of Cardiovascular Emergency, Internal Medicine and Geriatric, Medical School, Federico II University of Naples \\ ${ }^{2}$ General Direction, Medical School, Federico II University of Naples \\ ${ }^{3}$ Department of Translational Medical Sciences, Federico II University Naples
}

${ }^{\star}$ Corresponding author: Maurizio Santomauro, Department of Cardiovascular Emergency, Internal Medicine, Geriatricn Medical School, Federico II University, Via Sergio Pansini 5- 80131 Naples-Italy; Tel: 0039330 502275; E-mail: santomau@unina.it

Received: August 10, 2019; Accepted: August 30, 2019; Published: October 11, 2019;

\begin{abstract}
The Electrical Storm (ES) indicates cardiac electrical instability manifested by several episodes of ventricular tachyarrhythmias within a short time. False-ES is defined as recurrent inappropriate Implantable Cardioverter-Defibrillator (ICD) discharges over 24 hours. Far from being a minor complication, False-ES is usually physical and psychological harmful and potentially lethal. The most common causes of inappropriate ICD shock include supraventricular tachycardia with high ventricular response and oversening of peaked $\mathrm{T}$ waves or R wave, myopotentials or electrical noise. Appropriate diagnosis and treatment are critical in Emergency Department. To approach these patients systematically, it is important to understand that in general, there are four causes of shock. Modern ICD incorporate sophisticated tachycardia detection algorithms within their programming designed to minimize detection mistakes by the device and ICD-related information can also be checked using remote home monitoring systems. They are often not utilized to their full benefit. Thus, careful attention should be paid to the programming of the device. Fine tuning of the detection and differentiation algorithms is critical, and best done by a practitioner who understands the subtle differences among the different manufacturers. The approach to this problems is reviewed.
\end{abstract}

Keywords: False-Electrical Storm, Implanted cardioverter defibrillator, Inappropriate Shock

\section{Background}

Current definition of ES is the occurrence of three or more episodes of sustained VT or Ventricular fibrillation (VF) within 24 $\mathrm{h}$ requiring appropriate medical intervention. The same definition applies in ICD carriers in which ES is defined by three or more appropriate and separate (at least $5 \mathrm{~min}$ ) device interventions in 24 h, either with Antitachycardia Pacing (ATP) or shock [1]. Current guidelines recommend ICD implantation for secondary prevention of Sudden Cardiac Death (SCD) in survivors of cardiac arrest with no correctable causes and in patients with sustained symptomatic VT of different etiology. They also recommend ICD implantation for primary prevention in patients with ischemic or non-ischemic dilated cardiomyopathy and ejection fraction equal or lower than $35 \%$ after at least 3 months of optimized medical therapy [2] and in other less frequent inherited arrhythmogenic syndromes. For these reasons, ES is an increasingly frequent cause of access to Emergency Department (ED). It is estimated that about $25 \%$ of ICD carriers experience at least one ES episode per year follow-up [3,4]. Sometimes multiple recurrent ICD discharges are not associated with ES but are due to device malfunctioning. False-ES is defined as recurrent inappropriate ICD discharges over 24 hours. Far from being a minor complication, False-ES is usually physical and psychological harmful and potentially lethal. The most common causes of inappropriate ICD shock include supraventricular tachycardia with high ventricular response, device oversensing and mechanical malfunctions. Recurrent ICD shocks can cause myocardial injury by direct electrocution cell injury and by activation of signaling pathways in the molecular cascade of Heart Failure (HF), the most important of all are adrenergic neurohormonal system. Adrenergic iperactivity may then synergize with recurrent ventricular arrhythmias in exacerbating ventricular dysfunction and worsening HF. Sweeney et al. [5] demonstrated that electrical shocks were associated with an increased risk of death independently of underlying ventricular arrhythmia. Authors esteemes that for every delivered shock, whether appropriate or not, the risk of death increases by $20 \%$. On the other hand, no increased risk was associated with Antitachycardia Pacing (ATP) therapies. False-ES does not only cause myocardial damage, but can deplete a full device battery within hours, potentially leaving the patient unprotected from life-threatening arrhythmic events. False-ES should be treated by immediate intervention to suppress ICD shocks. Moreover, inappropriate discharges from ICD should be avoided at all cost by an optimal device programming [6].

\section{Implantable Device}

The ICD is a implantable device able to monitor cardiac rhythm and terminate potentially life-threatening arrhythmias. It consists of two main 
components: the generator that contains the battery, all the circuits that run the device, and the operator communicating system; the leads that reach heart chambers through the venous system and allow the device to monitor heart electrical activity and to deliver therapies. The ICD has a lead implanted in the right ventricle apex able to record ventricular activity and release therapies like pacing and/or direct current shock. In adjunct, some ICD has another lead implanted in the right atrium to record atrial electrical activity, improving discrimination between Supraventricular Arrhythmias (SVA) and ventricular arrhythmias and to pace the atrium (ICD-DR). ICD with Cardiac Resynchronization Therapy (CRT-D) has a third lead that paces the left ventricle (through the coronary venous system) synchronously to the right ventricle improving contractility. ICD uses mathematical algorithms defined by the manufacturer to discriminate life-threatening ventricular arrhythmias from supraventricular arrhythmias and to deliver appropriate therapy. Modern ICD stores information from various diagnostic features including intracardial ECG registrations during arrhythmia and can transmit these data using remote monitoring technology. Furthermore, the ICD can generate audible alarms in the case of device malfunction, low battery capacity and lead failure. Sometimes correct recognition fails and, in this case, the therapy delivered is defined inappropriate. In other cases the delivered therapy may not be able to terminate the ventricular arrhythmia, and it is defined ineffectiveVT recognition is primarily based upon tachycardia cycle length and duration. Both of these parameters are tailored on the patient's characteristics. Thus, ICD uses ventricular rate zones for rhythm classification. The boundaries between zones are defined by two main principles: the recognition of unstable fast VT/VF must be highly sensitive even at the cost of inappropriate rapid SVA treatment; the recognition of slower VT has to be more specific to avoid inappropriate therapies even at the cost of some delay in detection. The ICD treats ventricular tachyarrhythmias with two modalities: Antitachycardia Pacing (ATP) and Direct current shock. ATP is a brief ventricular pacing (6-8 beats) with a cycle length slightly lower (thus at a faster rate) of the arrhythmia, in the attempt of resetting the reentrant circuit and interrupting the arrhythmia; sometimes the paced cycle shortens from beat to beat and in this case it is referred as ATP ramp. Direct current shock is a biphasic electrical shock provided between the generator case and the coil localized on the right ventricular lead; the energy released may vary, reaching up to $41 \mathrm{~J}$ with the latest generation high-energy devices. Basing on several studies [8-19], ICD programming should empirically involve the use of three rate zones: a slow VT zone up to $320 \mathrm{~ms}$ cycle length ( $<188 \mathrm{bpm}$ ); a fast VT zone from 320 to 240 $\mathrm{ms}$ (188-250 bpm); a VF zone from $240 \mathrm{~ms}$ (>250 bpm). In VT zones a variable number of ATP attempts precedes the shocks delivery. In the slow VT zone, a greater number than in fast VT zone are usually programmed, as fast arrhythmias are usually less tolerated. In the VF zone, the hemodynamic instability of the arrhythmia and its high life-threatening potential require an immediate shock delivery. In modern devices an ATP during capacitor charging is delivered, avoiding the shock in the case of arrhythmic interruption. VT/VF detection isn't only based on ventricular rate but also requires a programmable duration of the arrhythmia to avoid detection of non-sustained episodes. Usually a VT/VF is detected when a certain percentage of ventricular sensed beats meets cycle length criteria.
The type of counting used varies between detection zones and between manufacturers. In order to improve sensibility, according to some manufacturers, the arrhythmia is detected when a certain percentage of beats falls in VF zone, while consecutive interval counting is required in the VT zone to increase specificity. The time to detection in the VT zone should be longer enough to allow spontaneous termination of non-sustained episodes.

\section{Inappropriate Therapies Due To Supraventricular Tachycardia}

Inappropriate therapies (especially shocks) are one of the main issues to be avoided because they cause patient discomfort, are potentially proarrhythmic and reduce battery life. The two main causes of inappropriate shock are failure in discriminating SVA and signal misinterpretation (Tab. 1) [11-20]. Frequently SVA are associated with a fast ventricular response leading ventricular rate to fall into VT/VF detection zone causing inappropriate therapy release. This problem occurs more frequently with single-chamber ICD that hasn't atrial sensing capabilities. Current guidelines don't provide a clear stepwise approach to managing patients at high risk for recurrent shock. Appropriate diagnosis and treatment are critical. Modern ICD incorporates sophisticated tachycardia detection algorithms inside their programming designed to minimize detection mistakes (Figures $1-5)$. Thus, careful attention should be paid the programming of the device. Fine tuning of the detection and differentiation algorithms is critical and best done by a practitioner who understands the subtle differences among the different manufacturers. Placing an atrial pacing lead and upgrading a single-chamber system to a dualchamber system for improved SVT discrimination is sometimes necessary and points out the significance of carefully screening for any history of SVT prior to initial ICD implant. ICD uses a variety of algorithms to discriminate SVA from VT. Major ones are listed below: -Atrio ventricular rate comparison : applies only in dual-chamber ICDs; when the ventricular rate is faster, the diagnosis is VT. When atrial and ventricular rates are equal, additional criteria are required for discrimination. -Onset: useful for discrimination of gradually accelerating sinus tachycardia from sudden-onset VT; it applies when the RR interval shortens by a programmed percentage if compared with the average number of preceding beats. May fail in case of VT occurring during sinus tachycardia. -Stability: useful for discrimination of fast response Atrial Fibrillation (AF). When RR interval variability is greater than a programmed percentage, $\mathrm{AF}$ is supposed. It may fail in the case of very fast $\mathrm{AF}$ in which there is a pseudo-regularization of ventricular rate, in atrial flutter or in irregular VT. -Morphology: it compares endocavitary electrocardiograms recorded during sinus rhythm and during VT. It is useful in single-chamber ICD lacking of atrial information, but may fail in intraventricular conduction delays and in rate-dependent conduction delays. -Rate duration: it is an extreme lifesaving measure. It results in shock delivery after a programmable time interval even if the episode is classified as SVA; this algorithm is usually activated when there is a high risk of undertreatment of VT erroneously recognized as SVA, but it increases the risk of overtreatment. 


\section{Biotronik: SMART}

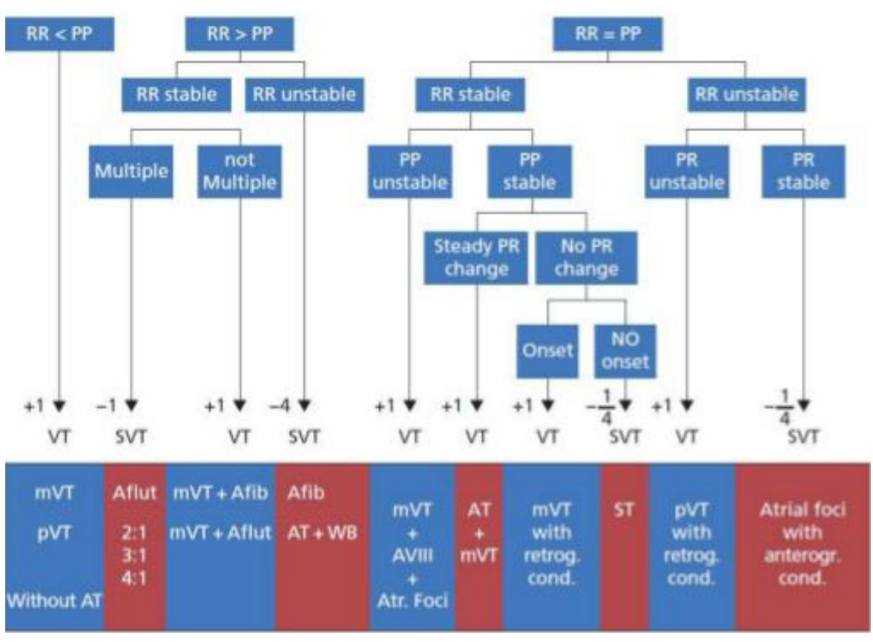

Figure 1. SMART Algorithm- based reduction of inappropriate defibrillation shock

\section{Boston scientific: RHYTHM ID}

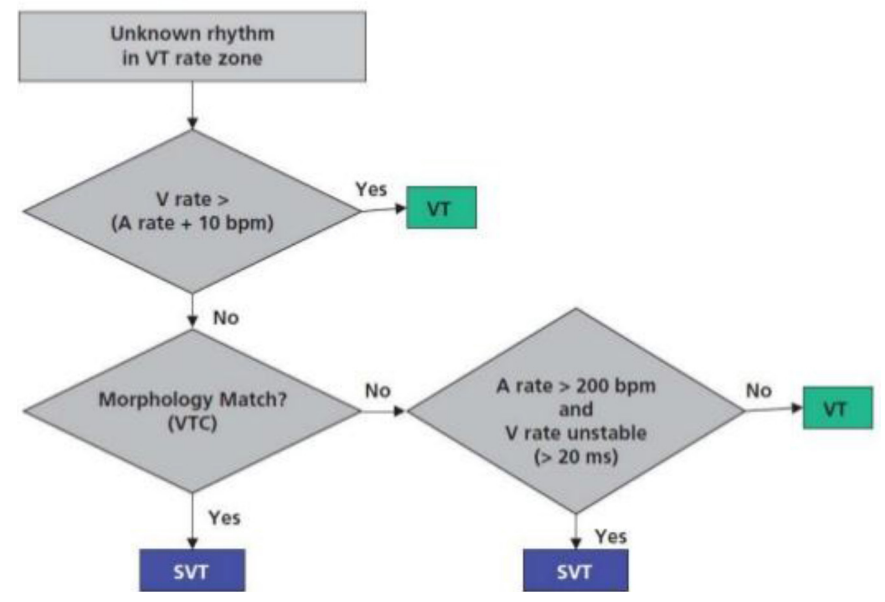

Figure 2. RHYTM ID Algorithm- based reduction of inappropriate defibrillation shock

\section{Medtronik: PR Logic}

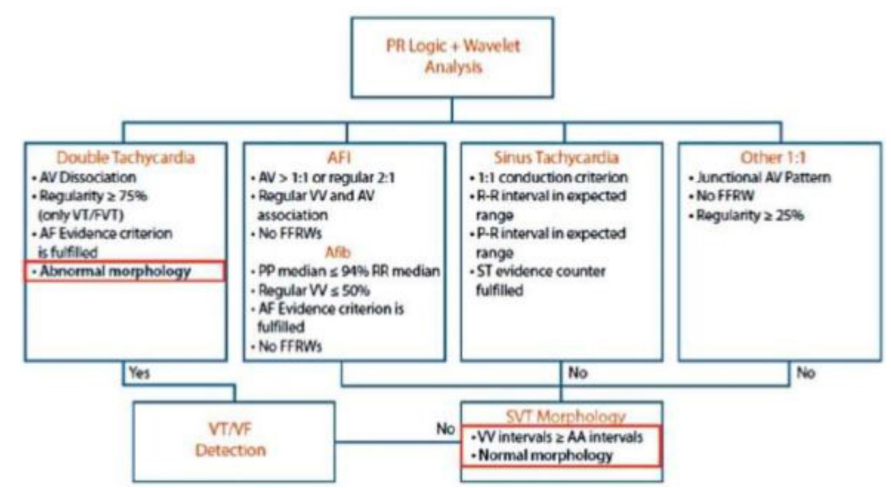

Figure 3. PR Logic Algorithm- based reduction of inappropriate defibrillation shock Sorin: PARAD+Rhythm DiScrimination

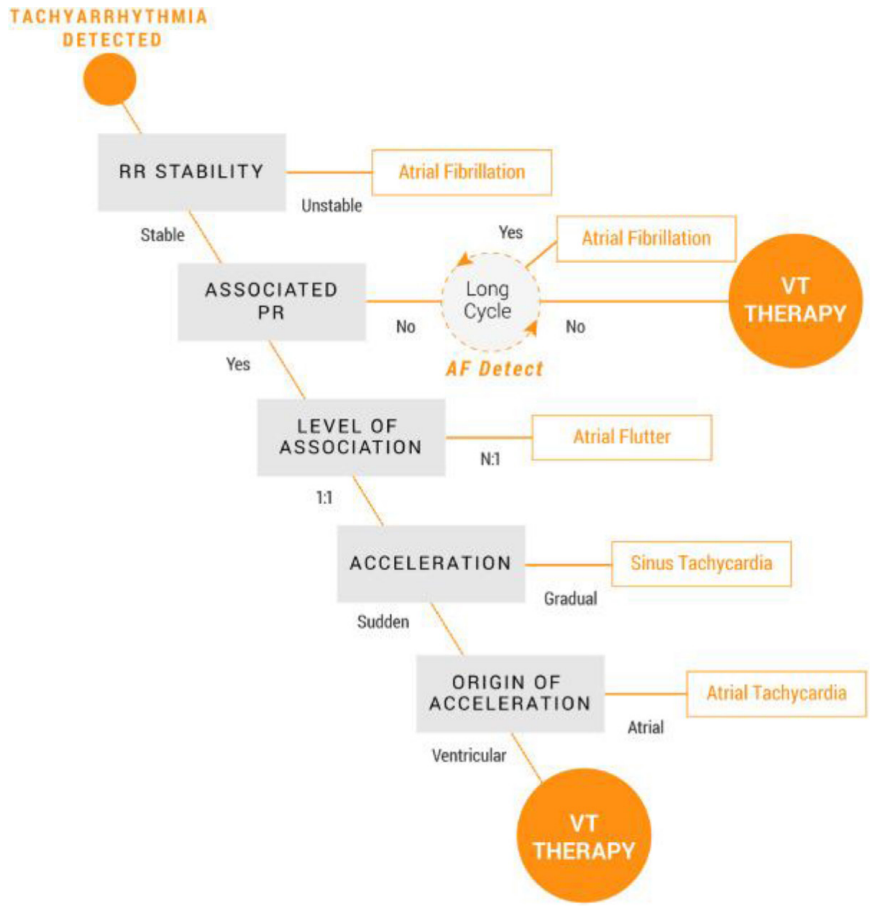

Figure 4. Sorin: PARAD+Rhythm DiScrimination Algorithm- based reduction of inappropriate defibrillation shock

\section{St Jude: Rate Branch}

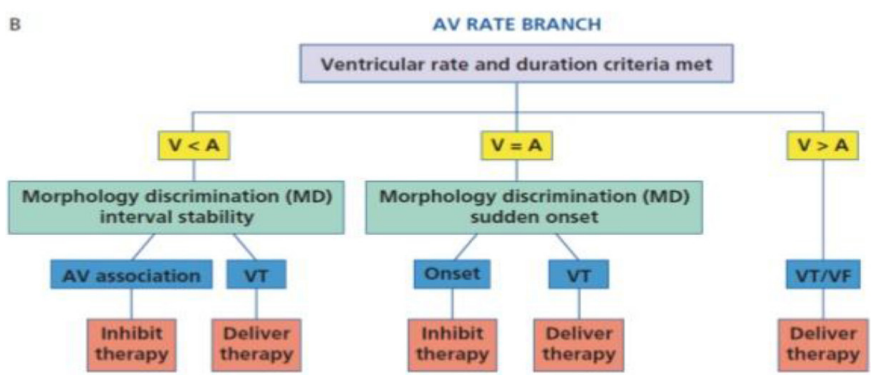

Figure 5. Rate Branch Algorithm- based reduction of inappropriate defibrillation shock

\section{Inappropriate Shock Due To Oversensing}

Signal misinterpretation is other big deal leading to inappropriate shocks. It may depend on some programmed easily editable variables and external and farfield interferences and lead fracture that usually requires an interventional approach [21-23] (Table 1). Major ones are listed below: $-\mathrm{T}$ wave oversensing: it happens when a high amplitude $\mathrm{T}$ wave is erroneously recognized as an $\mathrm{R}$ wave. It may happen because the low ventricular sensing threshold necessary to recognize even low-amplitude VF. This problem can be solved by increasing sensing threshold, lengthening refractory period or changing sensing decay parameters to suppress $\mathrm{T}$ wave detection. - Double-counted R waves: it may occur as a result of local ventricular delay in the baseline state or conduction delay caused by drugs or electrolyte abnormalities. It may also occur in patients with a double or triple lead ICD, long PR interval and loss of RV pacing capture. The ICD may count both the paced ventricular event and the spontaneous $\mathrm{R}$ wave conducted from the atrium. Finally, another common cause of double counting is loss of RV capture in CRT-Ds: the device counts both the paced ventricular 


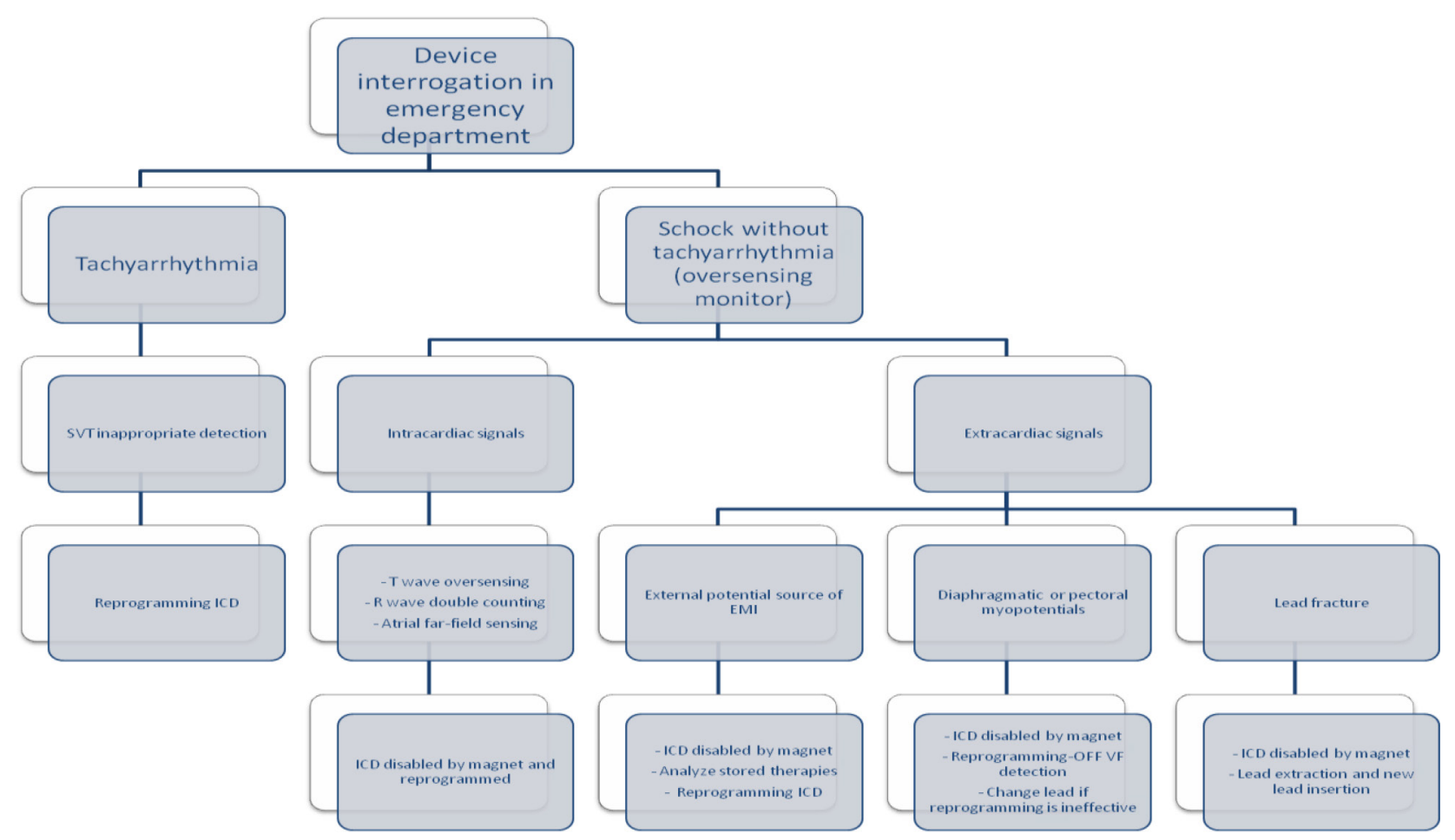

Figure 6. Stepwise algorithm for patients with frequent or repetitive ICD shocks

Table 1. Common Causes of Inappropriate Shock

\begin{tabular}{|c|}
\hline Supraventricular tachycardia with rapid ventricular response rate \\
\hline Atrial fibrillation \\
\hline Atrial flutter \\
\hline Atrial tachycardia \\
\hline Sinus tachycardia \\
\hline Device oversensing \\
\hline Intracardiac signals \\
\hline T-wave oversensing \\
\hline R-wave double counting \\
\hline Atrial far-field sensing \\
\hline Extracardiac signals \\
\hline Electromagnetic interference \\
\hline Pectoral myopotentials \\
\hline Diaphragmatic myopotentials \\
\hline Mechanical malfunctions \\
\hline Lead fracture \\
\hline Insulation break \\
\hline Lead dislodgement \\
\hline \\
\hline
\end{tabular}

event and the RV depolarization originating from the LV lead. R-wave double counting results in alternation of 2 ventricular cycle lengths. The second component of the $\mathrm{R}$ wave is usually sensed as soon as the blanking period terminates and is always classified in the VF zone. The classification of the first one depends on the programming of the tachy-zones and on the heart rate. The double counting can manifest during sinus rhythm, only during Precocious Ventricular Complex (PVC) or during slow VT with a misclassification as VF, the true rate being overestimated and possibly leading to shocks. Prolongation of the ventricular blanking period from the nominal value corrects ventricular double counting in the majority of cases and must be proposed as the first step when possible, keeping in mind that a common concern is true VF undersensing when the blanking period is over-extended. Similarly, decreasing the programmed ventricular sensitivity may resolve the problem in a certain number of cases but this option requires that reliable sensing of VF is confirmed at the reduced level of sensitivity. Moreover, lowering ventricular sensitivity may be dangerous and useless since the amplitude of the 2 signals may be as high. Programming of very high VF zone to solve the problem seems also inappropriate. Atrial far-field sensing: generated by inappropriately detecting an atrial paced event in the ventricular chamber related to the sensing of events from one chamber in another chamber. Cross-chamber blanking periods are an integral part of the ICD and CRT-D sensing systems. They are used to suppress detection of device-generated artifact as well as certain intrinsic signal artifacts. Events that occur during refractory and cross-chamber blanking periods are ignored for the purposes of pacing timing cycles and ventricular tachycardia detection. Each refractory and fixed crosschamber blanking period includes a re-triggerable noise window, which helps to detect and classify persistent noise. Cross-chamber blanking periods are designed to promote appropriate sensing of in chamber events and prevent oversensing of activity in another 
chamber. Cross-chamber blanking periods are initiated by paced and/or sensed events in an adjacent chamber. Residual energy on the defibrillation lead after shock delivery can increase the likelihood of cross-talk / far-field sensing. As this residual energy dissipates with time after shock delivery, the potential for cross-talk / far-field sensing also decreases. To reduce oversensing after shock delivery, a longer fixed value is automatically applied for all cross blanking periods during the Post-Therapy Period. -Electromagnetic Interference (EMI): is fortunately fairly infrequent with bipolar leads, but still occurs. There are many causes of EMI, the most common of which include Magnetic Resonance Imaging (MRI), large magnetic fields, arc welding, improper copper wiring in a shower, carrying stereo speakers, working on a running car engine, and lingering in a store's surveillance gating. To prevent shock from EMI often involves a certain amount of detective work. Once the cause of the EMI is identified, the patient must avoid the culprit, or in some cases, the device can be reprogrammed to prevent recognition of the EMI; -Pectoral Myopotentials: farfield myopotential recording may lead to inappropriate arrhythmic detection. This problem occurred in the past with unipolar leads using large sensing fields and is now largely avoided with the modern bipolar leads, recording more localized signals only. This highfrequency, variable amplitude signals are prominent on electrograms that include the ICD can, including shock electrograms and leadless ECG. They may be reproduced by pectoral muscle exercise. However, because ICD do not use these signals as primary sensing channels, pectoral myopotentials do not cause oversensing if the lead is intact. However, they may cause misclassification of exercise-induced sinus tachycardia as VT because algorithms that discriminate VT from SVT based on ventricular electrogram morphology use the RV coilcan vector as the default signal. Pectoral myopotentials might also interfere with algorithms that evaluate lead integrity by comparing near-field and far-field signals; -Diaphragmatic Myopotentials: these low-amplitude, high-frequency signals are more prominent on the sensing electrogram than the shock electrogram because the sensing bipole is closer to the source. Their amplitude varies with respiration, but not the cardiac cycle. Oversensing is most common with integrated bipolar sensing at the RV apex and rare with dedicated bipolar sensing or leads in the RV outflow tract. It occurs when sensitivity is maximal, after long diastolic intervals or ventricular paced events, and often ends with a sensed $\mathrm{R}$ wave, which reduces sensitivity abruptly. Thus, it commonly occurs in pacemaker-dependent patients, in whom inhibition of pacing maintains high ventricular sensitivity, resulting in persistent oversensing and inappropriate detection of VF. It may present as syncope because of inhibition of pacing followed by an inappropriate shock. With chronically implanted leads, oversensing may first occur after the dominant rhythm changes from ventricular sensed to ventricular paced, such as upgrade to CRT-D or AV junction ablation.Oversensing may be reproduced by monitoring real-time electrograms during deep breathing or straining in different positions, after programming VF detection off. -Lead failure: has many causes, but some of the most common include fractured leads, dislodged leads, loss of capture after ICD shock, redundant loops of endocardial leads, chatter in active fixation lead, loose set screew or adapter. Management of this category of shock involves fixing the implanted system, either with device reprogramming or reoperation. In these cases lead extraction and/or new lead insertion is the only choice. Modern devices usually provide alerts for lead integrity. The patient should be questioned about positional muscle twitching suggesting possible lead malfunction. If present, or if nonphysiologic noise is seen on the interrogation strips, active manipulation of the arm and device pocket should be performed while recording a rhythm strip with device channel markers through the interrogation box to determine if it is reproducible.

\section{Device Reprogramming}

Several studies demonstrated that repeated ICD shocks are associated with increased mortality as well as a reduction of quality of life [4]. For these reasons optimization of ICD programming in order to avoid unnecessary shock is mandatory in patients experiencing False-ES. As stated above, arrhythmic detection and treatment by ICD is a step process including several variables such as heart rate threshold, number of intervals to detect, discrimination of SVA, and type and number of therapies released. Each of these steps can be tailored upon patient characteristics to avoid unnecessary treatment. A patient who receives multiple shocks is not difficult to identify by ispecting data stored in the ICD. They will present to an ED with the specific complaint that their ICD has fired several times. At that point in time, it is critical to define the etiology of the shocks. Perform initial evaluation as above. The device needs to be fully interrogated, with careful analysis of all of the stored EGM recorded from the recent therapies and performing specific troubleshooting (Fig 6). The single most important diagnostic test is interrogation of the patient's device. If device malfunction is suspected, therapy (antitachycardia pacing and shock) can be immediately suspended by placing a magnet over the ICD can (Fig 6). Unlike a pacemaker, this will not alter the device's pacing capabilities. Should a true ventricular arrhythmia subsequently declare itself, removing the magnet will immediately reactivate all device therapies. Subsequent treatment will depend on the determined underlying cause. Device safety alerts are fortunately a reality and are more common with ICD than pacemakers. Prophylactic removal or replacement of a generator or lead on alert is generally not recommended unless the patient is pacer dependent. All device manufactures with products on alert have published management guidelines to physicians, which should be updated as new data is collected. The response to a safety alert must be individualized to each patient and balance the patient's risk of death from malfunction vs. the likelihood of malfunction and the known risk associated with going back in the pocket in terms of infection, perforation and bleeding.

\section{Conclusion}

The two main causes of False-ES are failure in discriminating SVA and signal misinterpretation. False-ES are a life-threatening syndrome and the appropriateness of acute management determines the patient's survival. Despite the difficulties associated with a comprehensive evaluation of this critical condition, a diagnostic approach based on the type of arrhythmia and the signals of device malfunction facilitates the mechanism-directed of inappropriate shocks. Recent advances in ICD reprogramming algorithm have greatly improved the clinical outcomes. 


\section{References}

1. Pedersen CT, Kay GN, Kalman J, Borggrefe M, Della-Bella P, et al. (2014) EHRA/ HRS/APHRS expert consensus on ventricular arrhythmias. Heart Rhythm 11: e16696Sep.

2. McMurray JJV, Adamopoulos S, Anker SD, Auricchio A, Böhm M, et al. (2012) ESC Guidelines for the diagnosis and treatment of acute and chronic heart failure 2012: The Task Force for the Diagnosis and Treatment of Acute and Chronic Heart Failure 2012 of the European Society of Cardiology. Developed in collaboration with the the Heart Failure Association (HFA) of ESC. Eur Heart J 33: 1787-847.

3. Santomauro M, Duilio C, Tecchia LB, Di Mauro P, Auricchio L, et al. (2010) Management of electrical storm in implantable cardioverter-defibrillator recipients. G Ital Cardiol 11: S37-S41.

4. Dorian P, Al-Khalidi HR, Hohnloser SH, Brum JM, Dunnmon PM, et al. (2008) Azimilide Reduces Emergency Department Visits and Hospitalizations in Patients With an Implantable Cardioverter Defibrillator in a Placebo-Controlled Clinical Trial. JACC 52: 1076-1083.

5. Sweeney MO, Sherfesee L, DeGroot PJ, Wathen MS, Wilkoff BL, et al. (2010) Differences in effects of electrical therapy type for ventricular arrhythmias on mortality in implantable cardioverter defibrillator patients. Heart Rhythm 7: 353360 .

6. Guerra F, Shkoza M, Flori M, Capucci (2012) A Electrical Storm. Cardiac Arrhythmias - New Considerations 2012: 377-394. Available : http://www. intechopen.com/books/cardiac-arrhythmias-new-considerations/electricalstorm

7. Wathen MS (2004) Prospective randomized multicenter trial of empirical antitachycardia pacing versus shocks for spontaneous rapid ventricular tachycardia in patients with implantable cardioverter- defi brillators: Pacing Fast Ventricular Tachycardia Reduces Shock Therapies (PainFREE Rx II) trial results. Circulation. 110: 2591-2596.

8. Cao J, Gillberg JM, Swerdlow CD (2012) A fully automatic, implantable cardioverter-defibrillator algorithm to prevent inappropriate detection of ventricular tachycardia or fibrillation due to T-wave oversensing in spontaneous rhythm. Heart Rhythm 9: 522-530.

9. Wilkoff BL, Williamson BD, Stern RS, Moore SL, Lu F, et al. (2008) Strategic programming of detection and therapy parameters in implantable cardioverterdefibrillators reduces shocks in primary prevention patients. $J$ Am Coll Cardiol. 52: 541-550.

10. Gunderson BD, Abeyratne AI, Olson WH, Swerdlow CD (2007) Effect of programmed number of intervals to detect ventricular fibrillation on implantable cardioverter-defi brillator aborted and unnecessary shocks. Pacing Clin Electrophysiol. 30: 157-165.

11. Tan VH, Wilton SB, Kuriachan V, Sumner GL, Exner DV (2014) Impact of programming strategies aimed at reducing nonessential implantable cardioverter defi brillator therapies on mortality: a systematic review and meta-analysis. Circ Arrhythm Electrophysiol 7: 164-170.

12. Gasparini M, Proclemer A, Klersy C, Kloppe A, Lunati M, et al. (2013) Effect of long-detection interval vs standard detection interval for implantable cardioverterdefi brillators on antitachycardia pacing and shock delivery: the ADVANCE III randomized clinical trial. JAMA 309: 1903-1911.

13. Martins RP, Blangy H, Muresan L, Freysz L, Groben L, et al. (2012) Safety and effi cacy of programming a high number of antitachycardia pacing attempts for fast ventricular tachycardia: a prospective study. Europace. 14: 1457-1464.

14. Madhavan M, Friedman PA (2013) Optimal programming of implantable cardiacdefibrillators. Circulation 128: 659-672.

15. Moss AJ, Schuger C, Beck CA, Brown MW, Josef Kautzner, et al. (2012) Reduction in Inappropriate Therapy and Mortality through ICD Programming. $N$ Engl $\mathrm{J}$ Med 367: 2275-2283.

16. Auricchio A, Schloss EJ, Kurita T, Meijer A, Steven Zweibel, et al. (2015) Low inappropriate shock rates in patients with single- and dual/triple-chamber implantable cardioverter-defibrillators using a novel suite of detection algorithms: PainFree SST trial primary results. Heart Rhythm. 12: 926-936.

17. Saeed M, Hanna I, Robotis D, Styperek R, Polosajian L, et al. (2014) Programming implantable cardioverter-defibrillators in patients with primary prevention indication to prolong time to first shock: results from the PROVIDE study. J Cardiovasc Electrophysiol. 25: 52-59.

18. Scott PA, Silberbauer J, McDonagh TA, Murgatroyd FD (2014) Impact of prolonged implantable cardioverter-defibrillator arrhythmia detection times on outcomes: a meta-analysis. Heart Rhythm 25: 52-59.

19. Peterson PN, Greenlee T, Go A S, Magid DJ, Cassidy-Bushrow A, et al. (2017) Comparison of Inappropriate Shocks and Other Health Outcomes Between Single- and Dual- Chamber Implantable Cardioverter- Defibrillators for Primary Prevention of Sudden Cardiac Death: Results From the Cardiovascular Research Network Longitudinal Study of Implantable Cardioverter- Defibrillators. $J$ Am Heart Assoc 6(11).

20. Ruiz-Granell R, Dovellini EV, Dompnier A, Khalighi K, García-Campo E, et al.
(2019) Algorithm-based reduction of inappropriate defibrillator shock: Results of the Inappropriate Shock Reduction wIth PARAD+ Rhythm DiScriminationImplantable Cardioverter Defibrillator Study. Heart Rhythm in press 16: 14291435 .

21. Swerdlow CD, Asirvatham SJ, Ellenbogen KA, Friedman PA(2014) Troubleshooting Implanted Cardioverter Defibrillator Sensing Problems I Circulation: Arrhythmia and Electrophysiology. 7:1237-1261.

22. Powell BD, Asirvatham SJ, Perschbacher DL, Jones PW, Cha YM, et al. (2012) Noise, artifact, and oversensing related inappropriate ICD shock evaluation: ALTITUDE noise study. Pacing Clin Electrophysiol 35: 863-869.

23. Mozes A, DeNofrio D, Pham DT, Homoud MK (2011) Inappropriate implantable cardioverter-defibrillator therapy due to electromagnetic interference in patient with a HeartWare HVAD left ventricular assist device. Heart Rhythm 8: 778-780.
Citation:

Maurizio Santomauro, Mario Petretta, Carla Riganti, Francesco Elia, Riccardo Franco, et al. (2019) Emergency in False-Electrical Storm in Patients with Implanted Cardioverter Defibrillator. J Cardiol Clin Pract Volume 2(1): 1-6. 